\title{
Etiology of Gingivitis
}

\author{
Antonio Bascones-Martínez, Elena Criado-Cámara, \\ Cristina Bascones-Ilundáin, Santiago Arias Herrera \\ and Jaime Bascones-Ilundáin \\ The Faculty of Dentistry, Complutense University, Madrid
} Spain

\section{Introduction}

Gingival diseases are a group of different disease entities that are localized to the gingiva. They all manifest clinical signs of inflammation and are classified into two main groups: plaque-induced and non-plaque-induced gingival diseases.

\section{Non-plaque-induced gingival diseases}

Gingival lesions not induced by plaque are common and can help explain the many different periodontal tissue reactions observed. Gingival inflammation sometimes differs from that of routine plaque-associated gingival diseases and often presents distinctive clinical features. (Holmstrup, 1999a).

The causes of non-plaque-induced gingival diseases include bacterial, viral, and fungal infections, genetic disorders and mucocutaneous diseases (e.g., lichen planus). Traumatic tooth brushing and allergic reactions to drugs are other possible causes.

\subsection{Gingival lesions associated with specific bacterial infections}

Bacterial infections can affect patients with and without immunodeficiency. Neisseria gonorrhoeae, Treponema pallidum, streptococci, Mycobacterium chelonae, are the most common bacterial infections that give rise to gingival lesions. They can manifest as fiery red, edematous, and painful ulcerations, asymptomatic chancres, mucous patches or atypical non-ulcerated, highly inflamed gingiva (Holmstrup, 1999b). These lesions may be associated with lesions on other parts of the body.

\subsection{Viral infections}

The most common viral infections are herpes simplex virus type1(HSV-1) and 2 (HSV-2) and varicella-zoster virus.

HSV is the most common viral infection of the oral/facial area. It has two subtypes: type 1, which affects the oral cavity; and type 2, which affects the genitals. Primary herpetic gingivo-stomatitis is most commonly observed in children from 7 months to 4 years of age but can also be found in adolescents or young adults. Children are often infected with HSV by their own parents if these have recurrent herpes lesions. The primary infection may be asymptomatic but can manifest as severe gingivostomatitis, in which the gingiva are 
painful, inflamed and ulcerated. Fever and lymphadenopathy are classic features and affected individuals experience difficulty in chewing.

The incubation period of the virus is 1 week, and healing occurs after approximately 10 to 14 days. Following infection and local replication at mucosal surfaces, HSV 1 enters sensory nerve endings and is transported by retrograde axonal transport to neuronal cell bodies, where a more restricted replication cycle takes place, usually culminating in the latent infection of these neurons. Latency allows maintenance of the viral genome in nonpathogenic and non-replicate form, serving as a reservoir for a later viral attack on the host. Reactivation of the virus in sensory ganglia causes cutaneous and mucocutaneous infection of the face, usually on the lips (Tovaru et al 2010). Reactivation of the virus is normally triggered by trauma, sun exposure, or menstrual periods, among other factors. These lesions are present in around $50 \%$ of the population and around $80 \%$ incubate the virus in latent form. Recurrent herpes infections can be found intra- and extra-orally. Intraoral herpes infection manifests as a group of painful ulcerations involving the gingiva and hard palate. The infection can be clinically diagnosed and confirmed by isolating the virus. HIV-positive individuals are more susceptible to viral infections and recurrence of herpetic lesions can be severe and potentially fatal in these patients.

Varicella-zoster virus causes chickenpox, primarily in children, and later reactivation of the virus in adults causes herpes zoster (shingles). Both can involve the gingiva, presenting as vesicle lesions that burst leaving fibrin-covered lesions.

This infection is readily diagnosed from the intense associated pain and unilateral lesions, which generally heal after 1-2 weeks. (Figure 1)

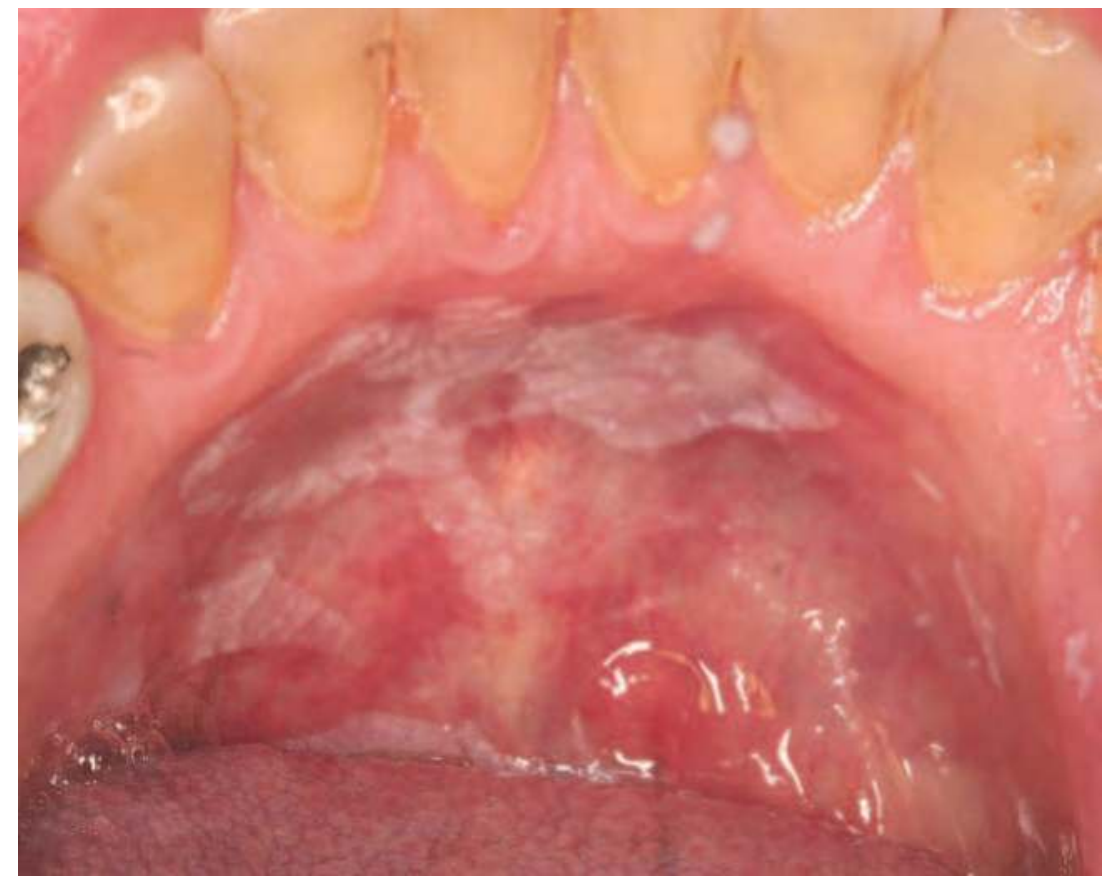

Fig. 1. HIV infection 


\subsection{Gingival lesions associated with fungal infections}

Gingival inflammation can also be caused by fungal infections such as candidosis, linear gingival erythema, and histoplasmosis.

\subsubsection{Candidosis}

Candida albicans is a diploid fungus (a form of yeast) that causes opportunistic oral and genital infections in humans. This commensal species populates the gastrointestinal tract. It affects $80 \%$ of the human population with no harmful effects but its overgrowth results in candidosis.

Infection by candida albicans is the most common mycotic infection of the oral mucosa and is commonly associated with impaired host immune responses such as:immunodeficiency, reduced salivary flow rate, smoking, corticosteroid treatment, or the use of antibiotiotics Candidosis is not usually observed in the gingival tissue of healthy individuals but, when affected, the most frequent clinical feature is gingival redness associated with a granular surface. This infection appears in four main forms: acute pseudomembranous candidosis, acute atrophic candidosis, chronic hyperplastic candidosis, and chronic atrophic candidosis. The diagnostic criteria for oral candidosis are: white plaques or diffuse erythematous areas, culture of C.albicans from saliva, presence of mycelium on direct examination of a smear from the lesion, biopsy showing hyphae in epithelium and characteristic histological changes, serum fluorescent antibody titer against Candida albicans $>1: 16$, and a positive antibody test with neat saliva. A further characteristic of primary oral candidosis is the alteration or disappearance of the lesion after treatment with antifungal agents (Holmstrup \& Axéll, 1990).

\subsubsection{Linear gingival erythema}

Linear gingival erythema is a non-plaque-induced gingival lesion exhibiting a distinct erythematous band of the marginal gingiva, with either diffuse or punctate erythema of the attached gingiva. It is characterized by intense gingival inflammation which does not respond to treatment with scaling and root planing or hygiene control. According to recent studies, the prevalence of linear gingival erythema ranges from 2 to $25 \%$ (Umadevi et al 2006).

\subsubsection{Histoplasmosis}

Histoplasmosis is caused by the fungus Histoplasma capsulatum, a soil saprophyte mainly found in the feces of cats and birds. H. capsulatum exists in mycelial form at room temperature and in yeast form at body temperature. Infection by $\mathrm{H}$. capsulatum usually occurs by inhalation of the spores and their deposition in the lungs. Most infections in the normal host are subclinical and asymptomatic.

There are three forms of histoplasmosis: a primary acute pulmonary form that is usually asymptomatic but may cause flu-like symptoms; a chronic pulmonary form, observed in a small percentage of patients, which is usually associated with underlying pulmonary disease; and a severe disseminated form, which is rare and observed in immunocompromised patients at the extremes of age or in otherwise debilitated patients. In recent years, histoplasmosis has joined other mycoses as a serious opportunistic infection associated with AIDS. 
Histoplasmosis in the head and neck is mainly seen in patients with disseminated disease and may be their only symptom. Oral lesions have been reported in $30 \%$ of patients with pulmonary histoplasmosis and $66 \%$ of patients with disseminated disease. They have been reported throughout the oral cavity, including gingiva, tongue, palate, buccal mucosa, oropharynx, retromolar area, lip, and floor of mouth. The lesions have been described as flat, plaque-like, non-tender elevations, either papillary or nodular, which subsequently ulcerate and become painful. Other descriptions include an erythematous, granulomatous appearance, a pattern of scattered red and white granulomatous lesions, and ulcerations as large as $2.5 \mathrm{~cm}$ in diameter. The definitive diagnosis is based on smear or culture and histology. (Stanford \& Rivera Hidalgo, 1999)

\subsection{Gingival lesions associated with genetic disorders}

Hereditary gingival fibromatosis is a very rare condition. It develops as an isolated disorder or as one feature of a syndrome; the most frequent characteristic is hypertrichosis;. Occasionally it is associated with mental retardation and epilepsy. The hyperplasic gingiva has a normal color and firm consistency with abundant stippling in adjacent gingiva. The buccal and lingual tissue of both mandible and maxilla may be involved, with interindividual variation in the degree of hyperplasia. Gingival fibromatosis can also be inherited as an autosomal dominant or recessive condition. The gingival enlargement usually begins with the eruption of permanent dentition. Gingival fibromatosis cannot be cured and usually involves the removal of large amounts of gingival tissue by conventional external bevel gingivectomy. (Ramer et al 1996)

\subsection{Gingival lesions associated with systemic conditions}

Systemic conditions that are associated with gingival inflammation include lichen planus, pemphigoid, pemphigoid vulgaris, multiform erythema, lupus erythema, drug-induced mucocutaneous diseases, and allergic reactions. Dermatologic diseases include not only numerous primary skin diseases but also common cutaneous manifestations of visceral or systemic diseases that may involve the oral mucosa. Dermatology is currently of major scientific and odontological interest, since oral lesions can be very early or even the only signs of various diseases (Gonçalves et al 2010). One of the main gingival disorders unrelated to plaque accumulation is desquamative gingivitis, which is characterized by epithelial desquamation, erythema, ulceration and/or vesiculobullous lesions in the gingiva and other epithelial tissues.

\subsubsection{Lichen planus}

Lichen planus is the most common mucocutaneous disease involving the gingiva, with a prevalence of $0.5-2.5 \%$. Some individuals present with lesions at other sites, such as the skin, while others are only affected in the oral cavity. The control and treatment of lichen planus is important, since it has premalignant potential. Its etiology is unknown. It manifests clinically in reticular or atrophic-erosive form, and the lesions show white papulae and striae, usually bilateral. Atrophic-erosive ulcerated lesions can sometimes be painful, whereas reticular forms have no significant symptoms. Lichen planus can affect any area of the oral mucosa, and its clinical appearance and extent can vary over the years, requiring differential diagnosis with other diseases such as leukoplakia. Subepithelial inflammatory reactions produced by lichen planus lesions are due to a specific unknown antigen, which is found at the point of attachment between the connective and epithelial tissues of the gum. (Holmstrup \& Dabelsteen, 1979). (Figure 2,3) 


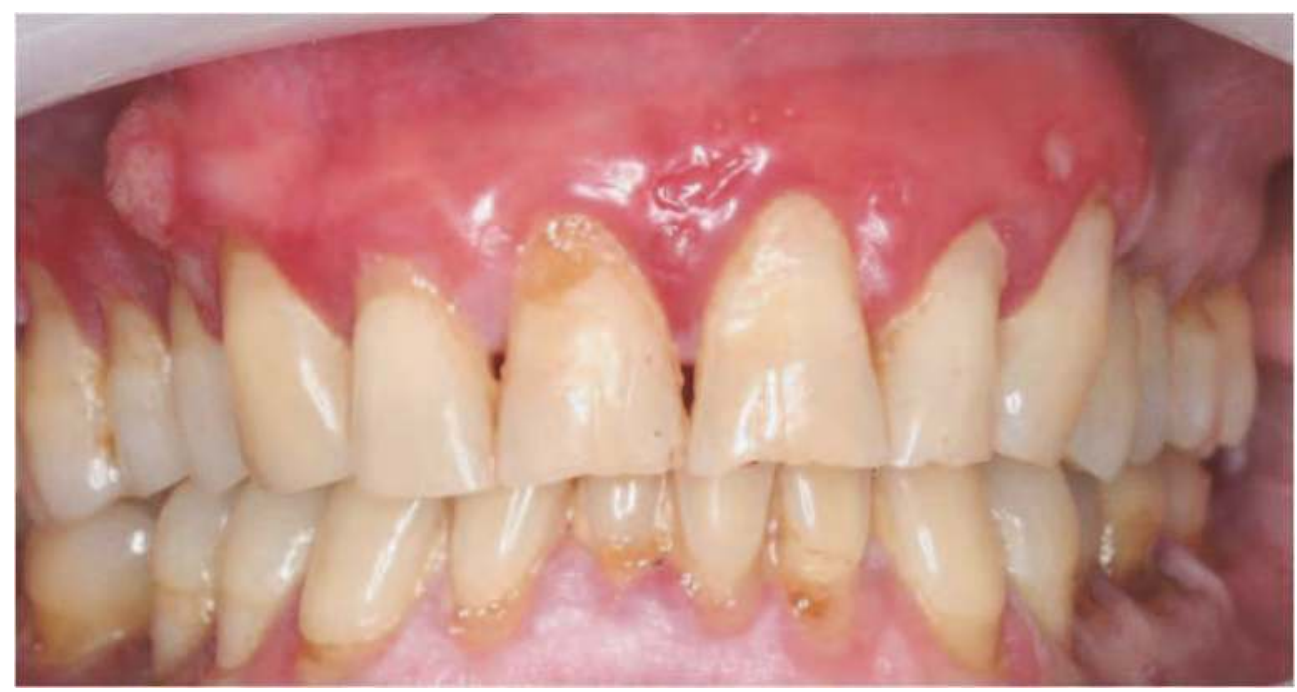

Fig. 2. Erosive formo of lichen planus

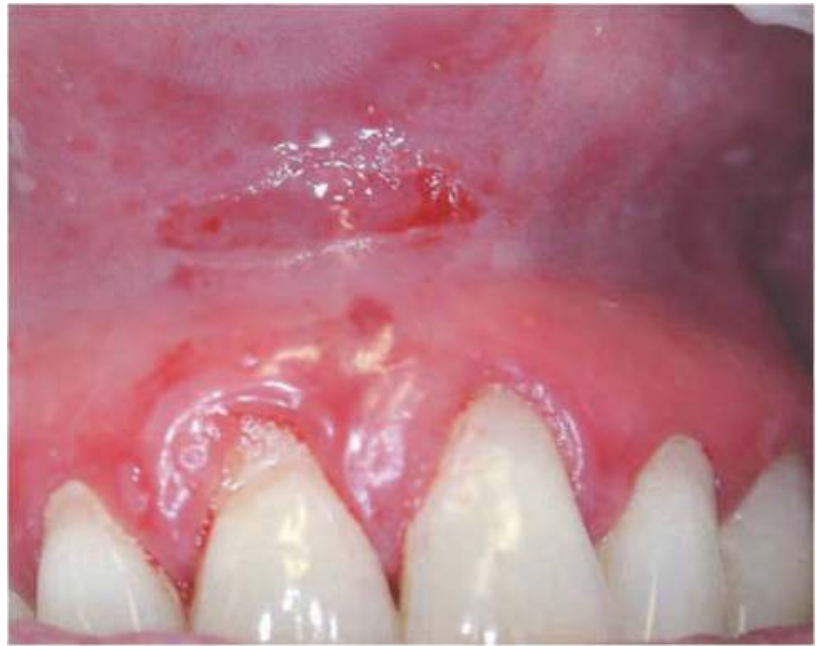

Fig. 3. Atrophic-erosive Lichen planus

Oral lichen planus is usually diagnosed by clinical and histological examinations, although the clinical appearance alone can suffice in classical lesions (bilateral white striae in cheek mucosa). The differential diagnosis includes lichenoid reactions to drugs or dental materials, leukoplakia, lupus erythematosus, and graft vs. host disease in bone marrow transplantation patients. Desquamative gingivitis may also be mistaken for pemphigus, pemphigoid, dermatitis herpetiformis, or linear IgA disease. Therefore, complementary examinations are essential in all cases to diagnose or rule out malignancy (Canto et al 2010).(Figure 4,5) 


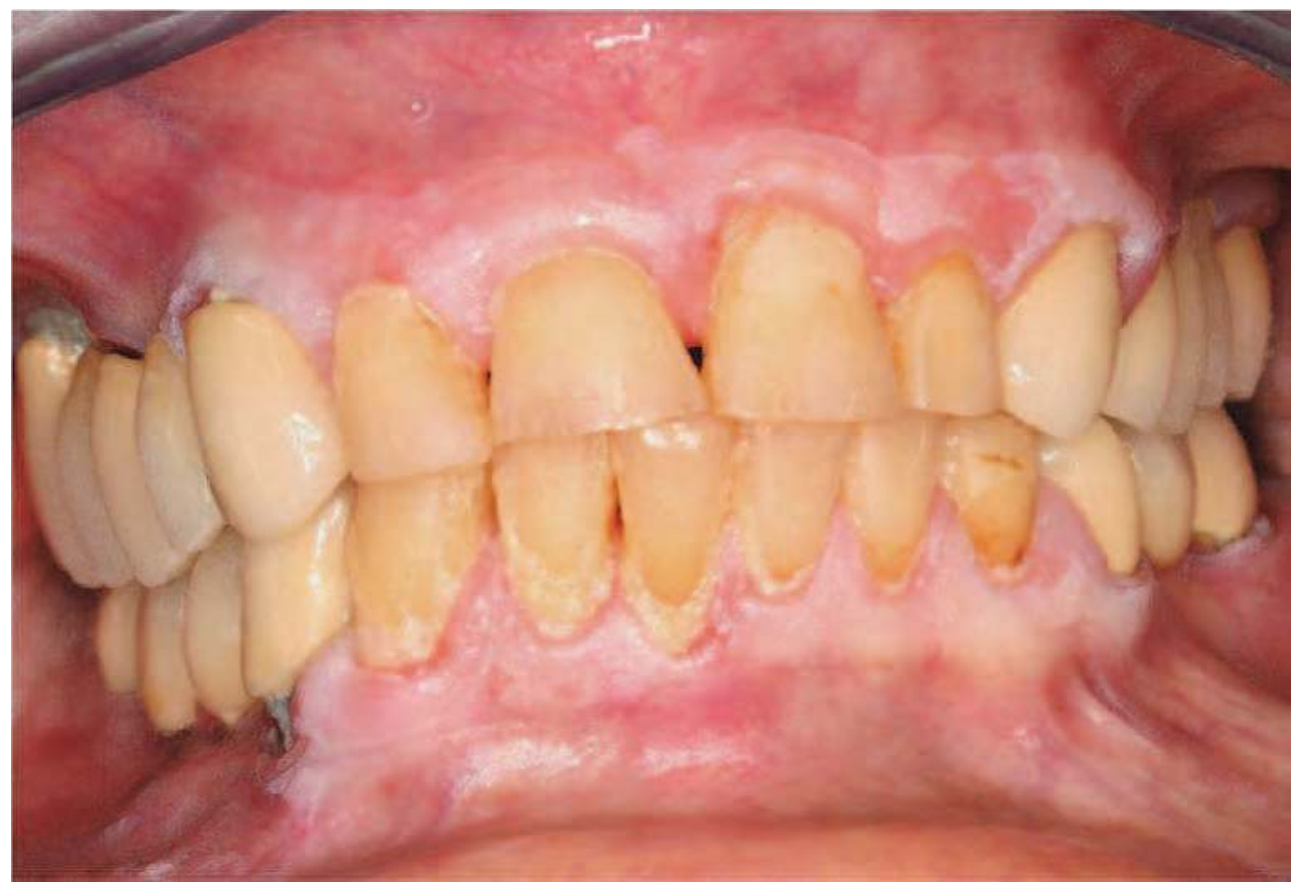

Fig. 4. Leukoplakia

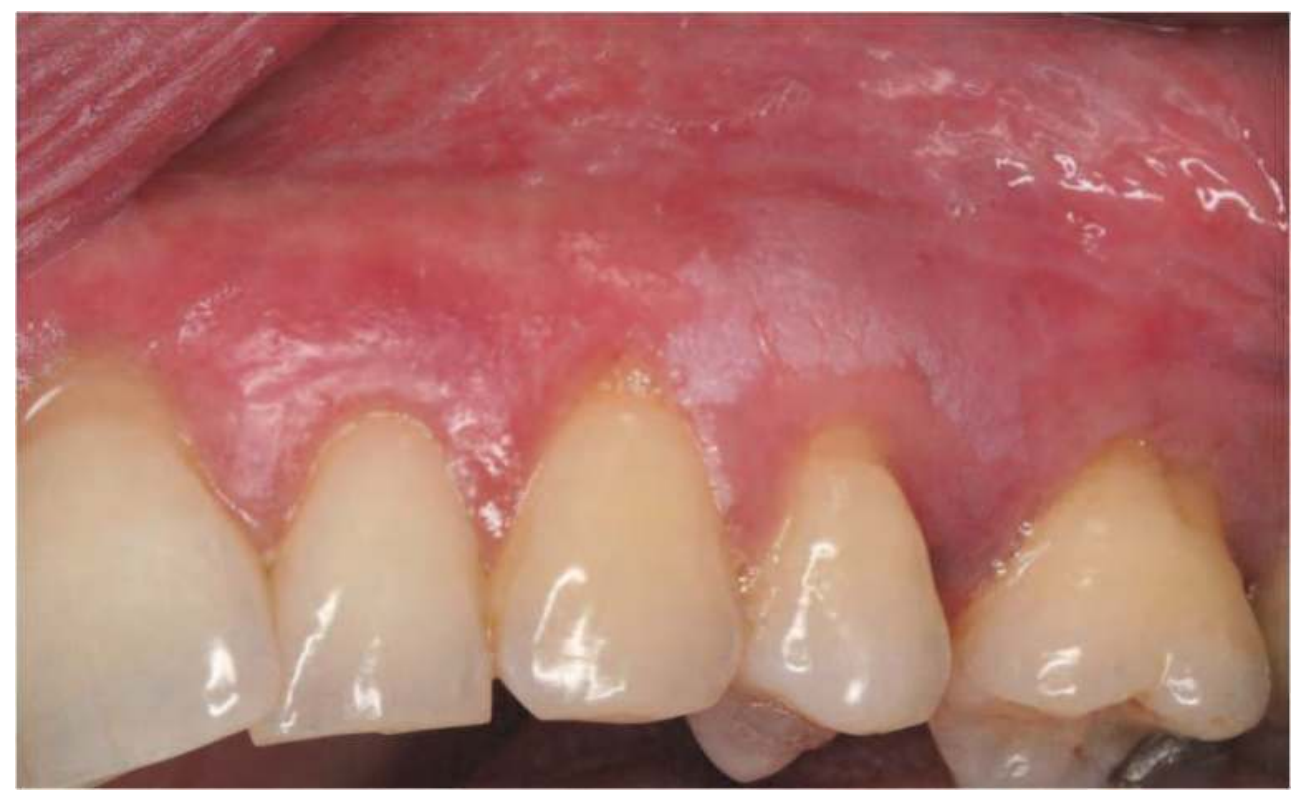

Fig. 5. Leukoplakia 


\subsubsection{Pemphigoid}

Pemphigoid is a group of disorders in which antibodies against the basal membrane components cause the epithelium to detach from the adjacent connective tissue. This condition normally appears in women over 50 years of age. It mainly affects the skin but can also affect the oral mucosa. Oral manifestations are almost inevitable, and the mouth is frequently the first affected site. The course of the disease is slow and progressive. Oral lesions (blisters) can be found in gingival tissue, buccal mucosa, and palate. These remain intact for the first 24-48 hours but finally burst, producing pain and stinging. (Nickolsky positive) Extraoral lesions on other sites are less common, although symblepharons are found on ocular mucosa and can lead to blindness if left untreated. (Figure 6)

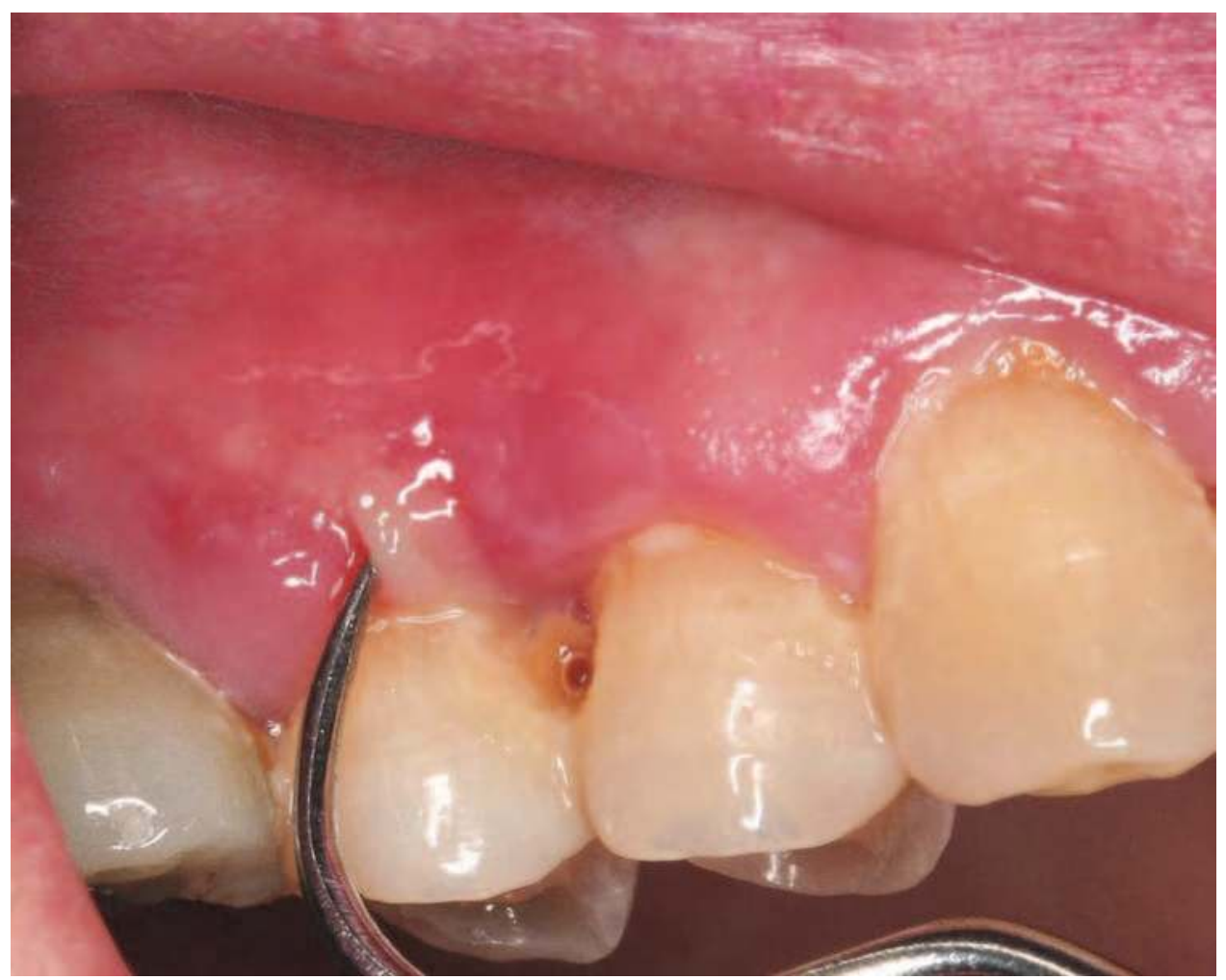

Fig. 6. Pemphigoid

\subsubsection{Pemphigus}

Pemphigus is group of autoimmune diseases characterized by the formation of blisters, due to loss of cohesion between keratinocytes in the epidermis caused by antibodies against desmoglein 1 and desmoglein 3. The mechanism by which the formation of antibodies is triggered remains unknown. Pemphigus vulgaris is the most common and severe form and is most frequent in the $6^{\text {th }}$ and $7^{\text {th }}$ decades of life. Its etiology appears to be genetic, while it is more frequent in individuals exposed to certain predisposing factors, including UV light and certain drugs. The blisters cover large areas of the skin and can be fatal if untreated. 
Individuals suspected of this condition must be referred immediately to a dermatologist. (Figure 7,8)

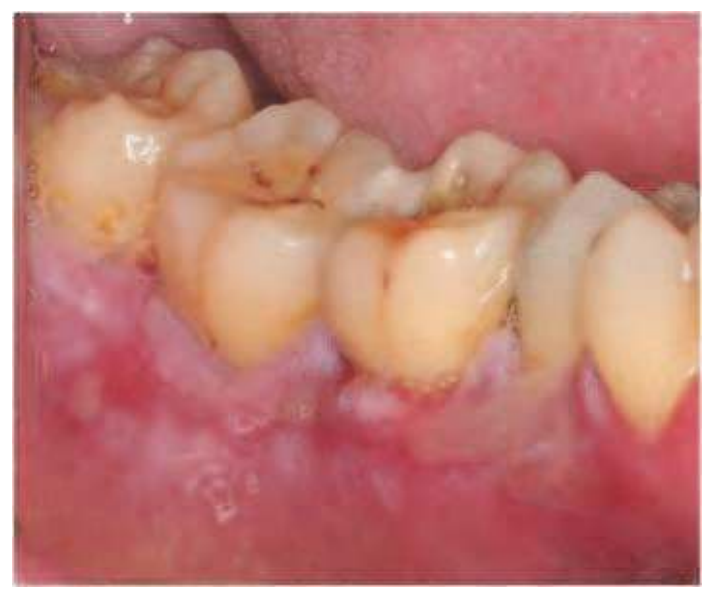

Fig. 7. Pemphigus

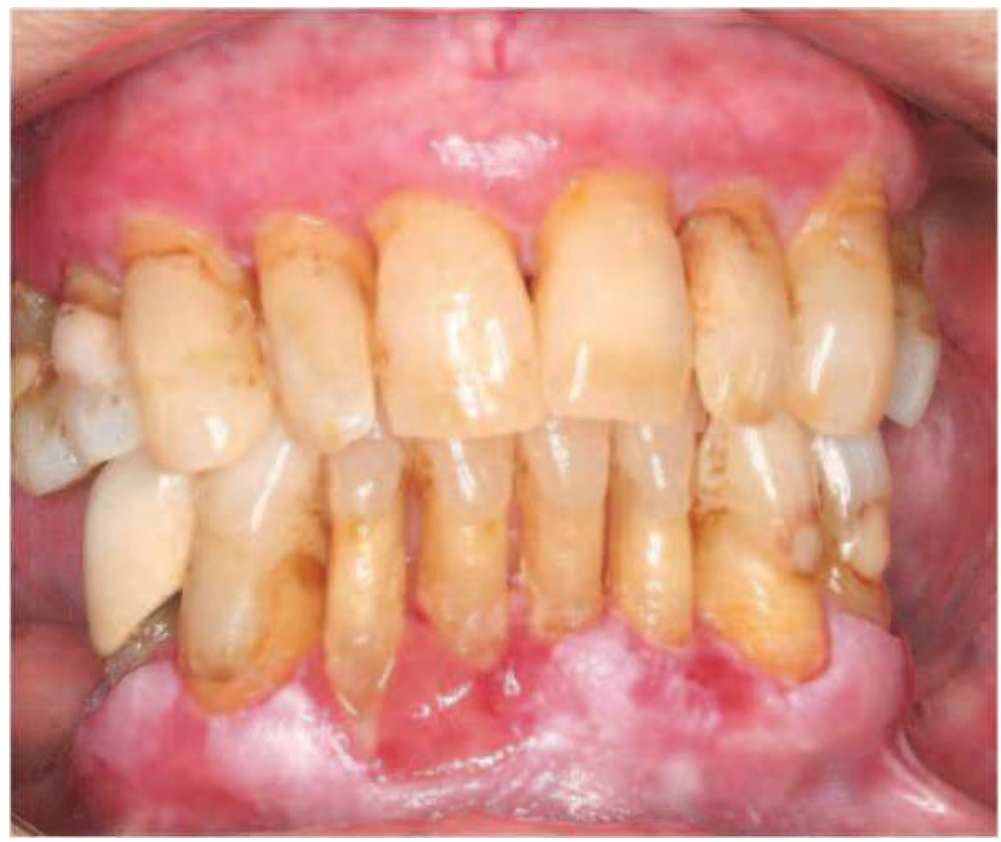

Fig. 8. Pemphigus

In the mouth, the lesions are localized in the gingival tissue and other sites. The blisters are small, fragile and asymptomatic until they burst, when they leave extremely painful and slightly bleeding erosions. The lesions contain non-adherent epithelial cells known as 
Tzanck cells. The treatment for pemphigus was decidedly unsatisfactory before the appearance of glucocorticosteroids in the 1950s, with mortality rates of $60-90 \%$. However, the high doses of glucocorticosteroids required to control pemphigus has led to a search for other agents with a higher therapeutic index. (Korman, 1988). The therapy currently consists of systemic corticosteroids and rigorous hygiene control.

\subsubsection{Erythema multiforme}

Erythema multiforme is a skin condition of unknown origin, possibly mediated by the deposition of immune complexes (mostly $\operatorname{IgM}$ ) in the superficial microvasculature of the skin and oral mucous membrane. It usually follows infection or drug exposure. It is a common disorder, with peak incidence in the $2^{\text {nd }}$ and $3^{\text {rd }}$ decades of life. Onset of this disease is usually preceded by general malaise.

The disease spectrum includes a mild and self-limited skin variant with exanthematic characteristics and minimal oral involvement and a severe, progressive variant that leads to extensive mucocutaneous epithelial necrosis, also known as Stevens-Johnson disease. In the milder form, oral lesions in the labial and buccal mucosa, tongue, palate, and gums change from papulae to blisters. Recurrence is very frequent, and the healing of lesions can take several weeks. Erythema multiforme has always posed a diagnostic challenge due to its extremely varied features, which can mimic other diseases. Nevertheless, the natural history and clinical and histological findings usually allow other conditions to be ruled out and a definitive diagnosis to be reached (Lozada \& Silverman, 1978). Treatment calls for corticosteroids and plaque control.(Figure 9).

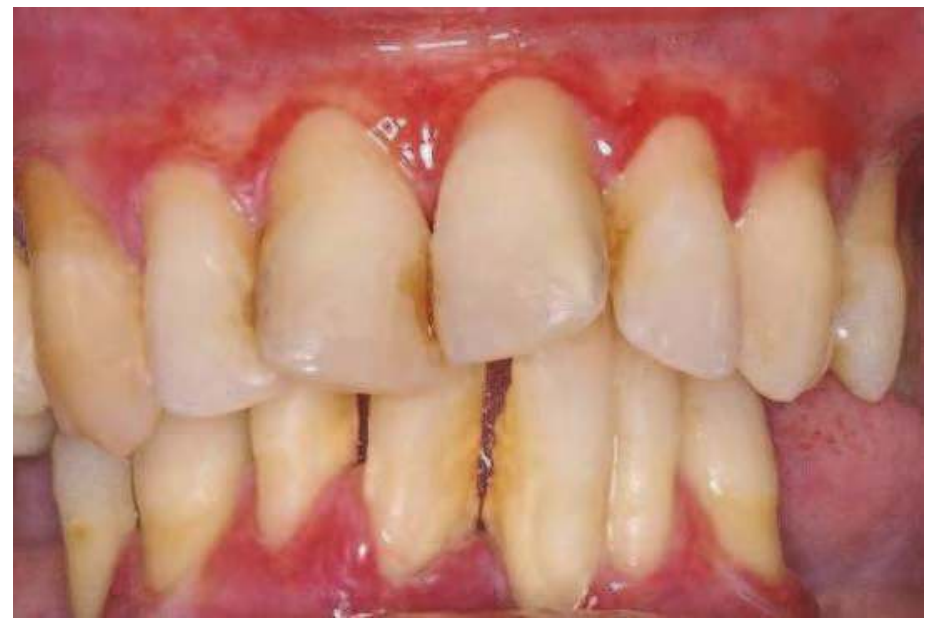

Fig. 9. Chronic desquamative gingivitis

\subsubsection{Lupus erythematosus}

Lupus erythematosus is a group of diseases in which anti-self antibodies are formed against various cell components, including the nucleus and cytoplasmic membrane. It more frequently affects females but shows no predilection for any anatomic site. The disease has two forms. One is systemic lupus erythematosus, which can affect mucosa, skin and other organs, including the kidneys and heart; the patients suffer from fever, weight loss, arthritis, 
and a typical butterfly-wing erythema on the cheekbone. The other, localized form is discoid lupus erythematosus, which only involves skin and mucosa. Orally, discoid lupus presents round erythematous lesions with a depressed central region surrounded by white striae, as well as less typical lesions, similar to Wickham striae, in the buccal mucosa, gingiva, and tongue. Treatment of the disorder includes topical corticosteroids alone or combined with immunosuppressants, and systemic corticosteroids in more severe cases.

\subsection{Drug induced gingival lesions}

Drug-induced mucocutaneous disorders can produce gingival hyperplasia, also known as gingival enlargement. Drug-induced gingival overgrowth is a side effect of three types of drugs: anticonvulsants (eg.Epanutin), immunosuppressive agents (eg. Cyclosporine), and various calcium channel blockers (e.g., Nifedipine) used for cardiovascular diseases. The overgrowth is characterized by the accumulation of extracellular matrix in gingival connective tissues. Recent studies suggested that these disorders may be induced by disruption of collagen synthesis homeostasis and gingival tissue degradation (Kataoka et al 2005a)

\subsection{Allergic reactions}

Oral manifestations of allergic reactions are uncommon. Reactions are mainly type I (immediate, mediated by IgE) or type IV (deferred, mediated by T cells). There are various possible causal agents, including materials used in dental procedures, oral hygiene products, chewing gum and foods. Materials such as mercury, gold, and acrylics can trigger type IV reactions, followed by the onset of white or erythematous lesions in the gingiva after 24-48 hours. Removal of the allergenic material is sufficient to stop the reaction. Dentifrices and mouthwashes can can cause edematous and red gingiva and affect the tongue. Foods that can potentially cause allergic reactions type I and IV include peanuts, kiwi fruit, and peaches.

\subsection{Other gingival manifestations of systemic diseases}

Other systemic diseases with gingival manifestations include gastrointestinal diseases (e.g., Crohn's disease), leukemia, and diabetes mellitus.

\subsubsection{Crohn's disease}

Crohn's disease, also known as regional enteritis, is an inflammatory disease of the intestines that can involve any part of the gastrointestinal tract, causing a wide variety of symptoms. It primarily causes abdominal pain, diarrhea (which may be bloody if the inflammation is severe), vomiting, or weight loss, but may also cause complications outside the gastrointestinal tract, such as rashes, arthritis, eye inflammation, tiredness, and lack of concentration. The oral lesions in Crohn's disease are similar to those of the gastrointestinal tract, including large ulcerations. The oral lesions are sometimes the first signs of the disease. Typical oral manifestations are folds in the labial or buccal sides of the sulcus.

\subsubsection{Leukemia}

Leukemia is a malignant haematologcal disorder characterized by an abnormal increase in white blood cells.. Leukemia is classified according to the course of the disease (acute or chronic) and the cell type involved. The main types are acute lymphoblastic leukemia, chronic lymphocytic leukemia, acute myelogenous leukemia, and chronic myelogenous 
leukemia. Oral manifestations have been reported in patients with acute monocytic leukemia, chronic myeloid leukemia, acute lymphocytic leukemia, and chronic lymphocytic leukemia. Gingival infiltration is the initial presenting complication in $5 \%$ of acute monocytic leukemia cases. Gingival infiltration of leukemic cells is most commonly seen in acute monocytic leukemia (M5) and acute myelomonocytic leukemia (Demirer et al 2007). Manifestations of leukemia include extended edema, ulceration, petechiae, and erythema. These are much more common in the acute than in the chronic form of the disease. Gingival edema in leukemic patients is mainly caused by plaque-induced inflammation, and plaque control is important. Gingival bleeding is also frequent in these patients due to secondary thrombocytopenia. Dental treatment is based on plaque control and, sometimes, on oral antibiotics administered one day before and one day after mechanical debridement.

\subsubsection{Diabetes mellitus}

Diabetes mellitus is characterized by disorders in insulin production, the metabolism of carbohydrates, fats, and proteins and in the functioning and structure of blood vessels. Patients with type I diabetes are at greater risk of developing gingivitis. Both children and adults with poor metabolic control show a greater tendency towards more severe gingivitis. The prevalence of gingivitis in children and adolescents with diabetes is nearly twice that observed in children and adolescents without this disease. The association between diabetes and gingivitis in children and adolescents is so widely accepted that diabetes mellitusassociated gingivitis appears as a specific entity in the most recent classification of periodontal diseases. Adults with type II diabetes may show higher rates of gingival inflammation versus adults without diabetes. Almost $64 \%$ of diabetics are estimated to have gingival inflammation, in comparison to $50 \%$ of non-diabetics. (Figure 10).

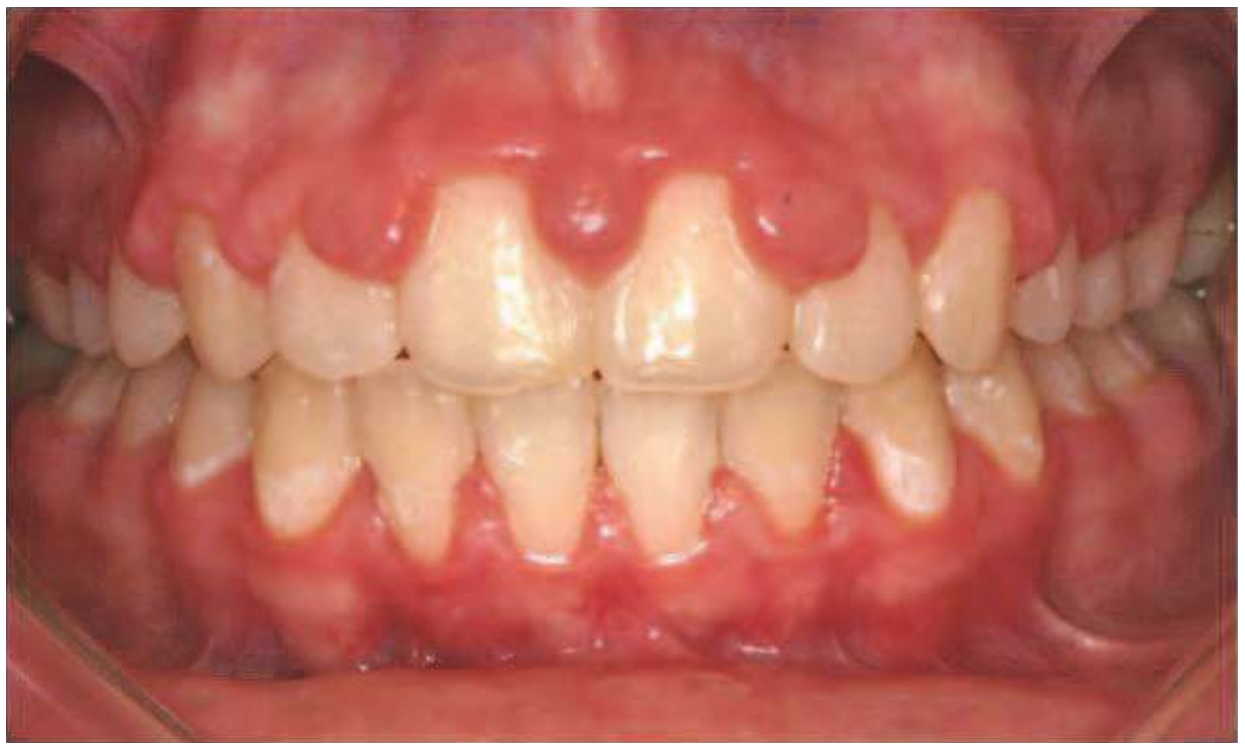

Fig. 10. Diabetes 
The degree of metabolic control in diabetes appears to be an important factor in the development and progression of gingivitis. Serum fructosamine levels, reflecting the patient's glycemic control over the preceding 2-3 weeks, are positively correlated with the degree of gingival bleeding and the severity of gingival inflammation in adults with type II and children with type I diabetes, respectively. The correlation between serum fructosamine levels and gingival inflammation in children was recently reported to persist into adolescence. Glycosylated hemoglobin $\left(\mathrm{HbA}_{1 \mathrm{c}}\right)$ values (reflecting blood glucose concentrations averaged over the preceding 6-8 weeks) above $10 \%$ (normal values $=4-6 \%$ ) appear to especially predispose children and adolescents to gingivitis. Notably, the presence of gingivitis in diabetic patients is not related to higher levels of plaque accumulation. The normalization of glycemic levels may significantly reduce the severity and extent of gingivitis in diabetic patients (Ryan et al 2003).

\subsection{Gingival lesions associated with trauma}

Injuries to oral soft-tissues can be produced by accidental, iatrogenic, and factitious traumas. Traumatic lesions, whether chemical, physical, or thermal, are relatively common in the mouth. Physical injury can also be self-inflicted (gingivitis artefacta), i.e., resulting from accidental trauma, premeditated infliction, or chronic habits, e.g., fingernail biting, digit sucking, or sucking on objects such as pens, pencils, or pacifiers (Dilsiz \& Aydin, 2009).

Physical trauma (e.g., from aggressive tooth brushing) can cause gingival lesions. Hyperkeratosis is the gingival response when the trauma is limited, whereas gingival laceration of the surface and tissue loss (gingival recession) can result from more violent traumas. Horizontal movement of the brush, abrasive dentifrices, and dental floss can also produce physical trauma of the gingiva. It is difficult to diagnosis these lesions by clinical evaluation, and the etiology cannot be identified in some cases.

Chemical injuries, such as those caused by chlorhexidine, are reversible and resolved by removal of the toxic substance.

Thermal injuries to the oral mucosa are commonly caused by hot drinks or food and most frequently affect the palate and labial mucosa. These lesions are painful, with an erythematous appearance, and may present vesicles, ulcerations or erosions of the mucosa.

Foreign bodies can also cause lesions in the oral cavity through the entrapment of materials, e.g., dental amalgam, in the gingival connective tissue. Amalgam pigmentation, generally called amalgam tattoo, is a relatively common finding in the oral mucosa. Tissue reaction to amalgam can vary considerably. It can arise as a macrophage or chronic inflammatory response, usually in the form of a foreign body reaction, or there can be no reaction (Santos Parizi \& Nai, 2010)

\section{Plaque-induced gingival diseases}

This group of gingival diseases are very prevalent and are initiated by dental plaque. The clinical features reflect the hosts's inflammatory and immune responses to the plaque bacteria. The clinical features of this condition include redness, swelling, and bleeding on probing. Other factors such as systemic disease, hormones, genetics, drugs, and malnutrition may influence the signs and symptoms of the disease. 
The classification of plaque-induced gingival diseases is based on the presence of dental plaque and the local or systemic factors that influence the level of gingival inflammation.

\subsection{Local modifying factors}

\subsubsection{Tooth anatomy}

\subsubsection{Tooth position}

The position or inclination of teeth can predispose the periodontium to plaque accumulation and subsequent inflammation. While studies show that areas of the periodontium adjacent to malaligned teeth can be maintained in a good state of health, periodontal disease can occur if meticulous oral hygiene is not practiced. In children, plaque and gingival inflammation scores have been correlated with malalignment.

\subsubsection{Root proximity}

Root proximity may present an impediment to self-performed or professionally applied plaque removal, increasing the risk of gingival inflammation.

\subsubsection{Open contacts}

Food impaction is likely if there are open contacts between the teeth. A significant relationship has been observed between food impaction and probing depth in a group of 40 healthy young male naval recruits, and the researchers concluded that food impaction contributes to periodontal disease.

\subsubsection{Root abnormalities}

Palato-gingival grooves are developmental abnormalities mainly observed in maxillary incisors. The presence of a groove from the crown that extends apically at the gingival margin can impede the removal of plaque and allow access of plaque microorganisms to the subgingival area. Proximal root groves can also be found on incisor teeth and maxillary premolars. These grooves have been associated with poor periodontal health, including attachment and bone loss, and they can appear on any tooth surface.

\subsubsection{Tooth restorations}

Poor restorations with ill fitting margins and orthodontic appliances can negatively influence the health of adjacent gingival tissues. It has been hypothesized that restorations violating the so-called "biological width" can produce an inflammatory response that may result in the loss of bone and connective tissue attachment and the migration of epithelial attachment. In most cases, the amount of damage to the periodontium is influenced by the severity and duration of the marginal discrepancy and the ability of patients to maintain the areas free of plaque.

\subsubsection{Effects of restorative materials}

Allergies to metals and acrylics commonly used in dental restorations have been reported. Damage to the periodontium can result from allergy to one or more of these materials (Blieden, 1999).

\subsection{Systemic modifying factors \\ 3.2.1 Endogenous hormones}

Periodontal tissues are modified by androgens, estrogens, and progestins. The homeostasis of periodontal tissues is a complex, multifactorial relationship that involves, at least in part, 
estrogen hormones. The intricate relationship between estrogen hormones and periodontal health has largely been studied in the gingiva. Clinical observations confirmed an increase in the prevalence of gingival disease with fluctuating plasma estrogen levels even when the oral hygiene remained unchanged. The etiology of estrogen-associated gingival diseases remains an enigma. Various authors have suggested that estrogens may modulate putative periodontal pathogens, blood vessels, and the immune system in the gingiva, but the influence of estrogen on these theoretical factors remains to be defined (Mariotti, 2005).

\subsubsection{Gingivitis associated with puberty}

The marked increase in steroid hormones in both sexes during puberty has a temporary effect on the inflammatory status of the gingiva. The signs of gingivitis in these cases are similar to those of classic plaque-induced gingivitis, although gingival inflammation can be found in adolescents with only a small amount of dental plaque accumulation.

\subsubsection{Gingivitis associated with the menstrual cycle}

Gingival tissues contain receptors for androgens, estrogens, and progesterone, which exert effects on the oral mucosa and periodontium. Changes in the circulating levels of female sex hormones also affect the host response against dental plaque (Becerik et al 2010). Women with gingivitis experience greater inflammation during ovulation with an associated increase in crevicular fluid exudate. Changes in gingival tissue during menstrual phases may be related to changes in inflammatory markers in the gingival crevicular fluid.

\subsubsection{Gingivitis associated with pregnancy}

The rise in hormone levels during pregnancy increases the risk of gingivitis, regardless of plaque levels. Various studies have found more gingival inflammation in pregnant than in postpartum women with the same amount of plaque. (Löe et al 1963). Pyogenic granuloma is an inflammatory hyperplasia that can be caused by hormonal factors; it appears on the gingiva as a smooth or lobulated exophytic lesion with small red erythematous papules on a pedunculated or sometimes sessile base, which is usually hemorrhagic and compressible. (Jafarzadeh et al 2006). This lesion is more frequent during the first trimester of pregnancy and normally disappears after giving birth.

\subsubsection{Gingivitis associated with drugs}

Gingival enlargement is a frequent side effect of certain drugs, including anticonvulsants (phenytoin, sodium valproate), immunosuppressive agents (cyclosporine A), and calcium channel blockers (nifedipine).

Phenytoin-induced lesion has aroused the curiosity of dental practitioners ever since its first description by Kimball in 1939, and interest has developed among cell biologists, connective tissue biochemists and geneticists over the past decade. However, intense research efforts have not elucidated the precise mechanism by which this simple molecule simultaneously prevents seizure activity in the brain while eliciting an adverse connective tissue reaction in the gums. For almost half a century, phenytoin was the only chemical compound regularly associated with a connective tissue response limited almost exclusively to the gingiva. However, phenytoin has been joined over the years by other systemic medications that have gingival overgrowth as an adverse effect. Although these new medicines are not structurally related in any way to phenytoin, they elicit gingival manifestations with an uncanny clinical and microscopic resemblance to the gingival enlargement associated with phenytoin (Hassel \& Hefti 1991). 
Reported prevalence rates vary widely from 10 to $50 \%$ for phenytoin, 8 to $70 \%$ for cyclosporine A, and 0.5 to $83 \%$ for nifedipine. The presence of various degrees of gingival inflammation hampers the accurate assessment of drug-induced gingival overgrowth per se, which is exacerbated by the inflammation (Kataoka et al 2005b).

\subsubsection{Anticonvulsants (Phenytoin)}

Phenytoin is an anticonvulsant used in the treatment of epilepsy. Approximately $40-50 \%$ of all individuals treated with phenytoin develop esthetically disfiguring enlargement of the gingiva. The lesion is histologically characterized by an accumulation of the connective tissue matrix within the gingiva propria. Although the pathogenesis is multifactorial, there is a consensus that direct action of phenytoin upon resident gingival fibroblasts is the primary causative factor (Hassel, 1983).

\subsubsection{Immunosuppressive agents (Cyclosporine A)}

This drug prevents the rejection of transplanted solid organs and bone marrow. Despite its unequivocal success as a truly selective immunosuppressive drug, cyclosporine $\mathrm{A}$ is also associated with gingival enlargement.(Hassel \& Hefti, 1991), with a prevalence ranging from 6 to $80 \%$. It appears as a progressive gingival growth that starts with a more intense enlargement of interdental papillae in the anterior area of the mouth. The presence of dental plaque plays an important role in worsening the clinical picture.

\subsubsection{Calcium channel blockers (Nifedipine)}

In clinical use since 1977 for the treatment and prophylaxis of acute and chronic coronary insufficiency, nifedipine was first reported in 1984 to be causatively associated with gingival enlargement (Hassel \& Hefti, 1991). The prevalence of gingival overgrowth in treated patients ranges from 15 to $25 \%$. These drugs selectively block the entrance of calcium channels at cell level, hence increasing the extracellular calcium count. The enlarged gingiva is firm in these cases, with no tendency to bleed, and is normally more prominent in the anterior vestibular area.

\subsubsection{Gingivitis associated with oral contraceptives}

Oral contraceptives were first approved in the USA in 1960 and are currently used by almost 12 million women in the USA and more than 100 million women worldwide. Usage varies widely by country, age, education and marital status. It has been suggested that hormonal contraceptive use by women of childbearing age increases their risk of periodontal disease. Early small-scale clinical studies found that combined oral contraceptives containing high doses of estrogen increased the risk of gingival disease, suggesting an adverse effect on the underlying supporting periodontal tissues. Other studies reported that gingival inflammation increased in direct relationship with the duration of combined oral contraceptive use. Limited animal studies also have lent support to these notions, indicating that oral contraceptives have marked effects on the gingival microvasculature, altering capillary permeability and producing the cell immune response seen in gingival diseases. As a result of these data, the dental community has often attributed poor gingival health to the use of oral contraceptives, despite these studies being more than 25 years old.

A recent small-scale clinical study found no association between the use of low-dose oral contraceptives and gingivitis, but the relationship between OCs and periodontal diseases has yet to be investigated in a large representative population-based sample. A crosssectional study in premenopausal women found no association between previous high-dose 
or current low-dose oral contraceptive intake and increased levels of gingivitis or periodontitis, emphasizing the need for a reexamination of the perceived association between these drugs and periodontal diseases (Taichman \& Eklund, 2005).

\subsubsection{Gingivitis associated with malnutrition}

Severe periodontal disease, accompanied by gingival hemorrhage, tooth mobility and attachment loss, was traditionally considered a clinical feature of ascorbic acid deficiency. However, it has been suggested that various forms of gingivitis and periodontitis mainly result from the activities of oral microorganisms that colonize the teeth and adjacent periodontal tissues, assigning a secondary role to ascorbic acid deficiency; in fact, most of the epidemiological and experimental evidence accumulated over recent decades has failed to demonstrate any significant etiological relationship between ascorbic acid deficiency and periodontal disease (Leggot et al 1991).

\subsubsection{Gingivitis associated with ulcerated lesions}

Necrotizing gingivitis (NG) or necrotizing ulcerative gingivitis (NUG) is an acute opportunistic gingival infection caused by bacterial plaque. It appears more frequently in undernourished children and young adults and in immunodeficient individuals. The disease is characterized by pain, bleeding, and papillary necrosis and has a tendency to relapse (Bermejo Fenoll \& Sanchez Pérez 2004). Its prevalence is fairly low $(<0.5 \%$ in industrialized countries), although an increase has recently been observed among young adults in relation to smoking, stress and other factors. HIV-positive individuals are also more susceptible to necrotizing periodontal disease, with a reported prevalence ranging from $0 \%$ to $11 \%$.

\section{Conclusions}

Gingivitis can have multiple origins and can be the manifestation of a wide range of systemic diseases. Gingival tissue inflammation is one of the most common lesions encountered in the clinical setting and may be the first symptom in many types of disease. Gingivitis may therefore have important diagnostic relevance, and it is vital for clinicians to be aware its different possible causes to ensure a correct diagnosis and treatment.

\section{References}

Becerik S, Ozçaka O, Nalbantsoy A, Atilla G, Celec P, Behuliak M, Emingil G. (2010). Effects of menstrual cycle on periodontal health and gingival crevicular fluid markers. J Periodontol. 2010 May;81(5):673-81.

Bermejo-Fenoll A, Sánchez-Pérez A. (2004) Necrotising periodontal diseases. Med Oral Patol Oral Cir Bucal. 2004;9 Suppl:114-9; 108-14

Blieden TM. Tooth related issues. Ann Periodontol. 1999 Dec;4(1):91-7.

Canto AM, Müller H, Freitas RR, Santos PS. (2010) Oral lichen planus (OLP): clinical and complementary diagnosis. An Bras Dermatol. 2010 Oct;85(5):669-75. 
Demirer S, Ozdemir H, Sencan M, Marakoglu I. (2007) Gingival hyperplasia as an early diagnostic oral manifestation in acute monocytic leukemia: a case report. Eur J Dent. 2007 Apr; 1(2):111-4.

Dilsiz A, Aydin T. (2009) Self-inflicted gingival injury due to habitual fingernail scratching: a case report with a 1-year follow up. Eur J Dent. 2009 Apr;3(2):150-4.

Hassell TM, Gilbert GH. (1983) Phenytoin sensitivity of fibroblasts as the basis for susceptibility to gingival enlargement. Am J Pathol. 1983 Aug;112(2):218-23.

Hassell TM, Hefti AF. (1991) Drug-induced gingival overgrowth: old problem, new problem. Crit Rev Oral Biol Med. 1991;2(1):103-37.

Holmstrup P, Dabelsteen E. (1979)Changes in carbohydrate expression of lichen planus affected oral epithelial cell membranes. J Invest Dermatol. 1979 Nov;73(5):364-7.

Holmstrup P, Axéll T. (1990) Classification and clinical manifestations of oral yeast infections. Acta Odontol Scand. 1990 Feb;48(1):57-9.

Holmstrup P. (1999) Non-plaque-induced gingival lesions. Ann Periodontol. 1999 Dec;4(1):20-31.

Jafarzadeh H, Sanatkhani M, Mohtasham N. (2006) Oral pyogenic granuloma: a review. J Oral Sci. 2006 Dec;48(4):167-75.

Kataoka M, Kido J, Shinohara Y, Nagata (2005) T. Drug-induced gingival overgrowth--a review. Biol Pharm Bull. 2005 Oct;28(10):1817-21.

Korman N.(1988) Phempigus. J Am Acad Dermatol. 1988 Jun;18(6):1219-38.

Leggott PJ, Robertson PB, Jacob RA, Zambon JJ, Walsh M, Armitage GC. (1991) Effects of ascorbic acid depletion and supplementation on periodontal health and subgingival microflora in humans. J Dent Res. 1991 Dec;70(12):1531-6.

Lindhe, J. ( 2009) Periodontología clínica e implantología odontológica. $5^{\circ}$ ed. Panamericana ISBN 978-950-06-1457-3 Buenos Aires.

Loe H, Theilade E, Jensen SB. (1965) Experimental gingivitis in man. J Periodontol. 1965 May-Jun;36: 177-87.

Loe H, Silness J. (1963)Periodontal disease in pregnancy. I. Prevalence and severity. Acta Odontol Scand. 1963 Dec; 21:533-51.

Lozada F, Silverman S Jr. (1978) Erythema multiforme. Clinical characteristics and natural history in fifty patients. Oral Surg Oral Med Oral Pathol. 1978 Nov;46(5):628-36.

Gonçalves LM, Bezerra Júnior JR, Cruz MC. (2010) Clinical evaluation of oral lesions associated with dermatologic diseases. An Bras Dermatol. 2010 Apr;85(2):150-6.

Mariotti A. (1999) Dental plaque-induced gingival diseases. Ann Periodontol. 1999 Dec;4(1):7-19.

Mariotti A. (2005) Estrogen and extracellular matrix influence human gingival fibroblast proliferation and protein production. J Periodontol. 2005 Aug;76(8):1391-7.

Ramer M, Marrone J, Stahl B, Burakoff R. (1996) Hereditary gingival fibromatosis: identification, treatment, control. J Am Dent Assoc. 1996 Apr;127(4):493-5.

Ryan ME, Carnu O, Kamer A. (2003) The influence of diabetes on periodontal tissues. J Am Dent Assoc. 2003 Oct; 134 Spec No:34S-40S.

Parizi JL, Nai GA. (2010) Amalgam tattoo: a cause of sinusitis? J Appl Oral Sci. 2010 Jan-Feb; 18(1):100-4.

Stanford TW, Rivera-Hidalgo F. (2000) Oral mucosal lesions caused by infective microorganisms. II. Fungi and parasites. Periodontol 2000. 1999 Oct;21:125-44.

Taichman LS, Eklund SA. (2005).Oral contraceptives and periodontal diseases: rethinking the association based upon analysis of National Health and Nutrition Examination Survey data. J Periodontol. 2005 Aug;76(8):1374-85. 
Tovaru S, Parlatescu I, Tovaru M, Cionca L, Arduino PG. (2010) Recurrent intraoral HSV-1 infection: a retrospective study of 58 immunocompetent patients from Eastern Europe. Med Oral Patol Oral Cir Bucal. 2010 Aug 15.

Umadevi M, Adeyemi O, Patel M, Reichart PA, Robinson PG. (2006) (B2) Periodontal diseases and other bacterial infections. Adv Dent Res. 2006 Apr 1;19(1):139-45 


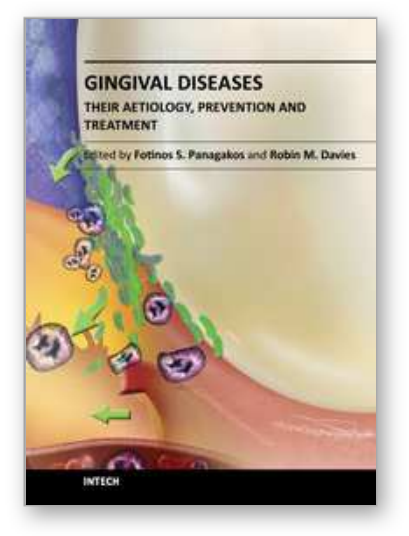

\author{
Gingival Diseases - Their Aetiology, Prevention and Treatment \\ Edited by Dr. Fotinos Panagakos
}

ISBN 978-953-307-376-7

Hard cover, 230 pages

Publisher InTech

Published online 22, September, 2011

Published in print edition September, 2011

Gingival diseases are a family of distinct pathological entities that involve the gingival tissues. These signs and symptoms of these diseases are so prevalent in populations around the world that they are often considered to be â€œnormalâ€ features. The diseases are now classified into two main groups namely: Plaque-Induced and Non-Plaque Induced Gingival Diseases. This book provides dentists, dental hygienists, dental therapists and students with a comprehensive review of gingival diseases, their aetiology and treatment.

\title{
How to reference
}

In order to correctly reference this scholarly work, feel free to copy and paste the following:

Antonio Bascones-Martinez, Elena Criado-Cámara, Cristina Bascones-llundáin, Santiago Arias Herrera and Jaime Bascones-Ilundáin (2011). Etiology of Gingivitis, Gingival Diseases - Their Aetiology, Prevention and Treatment, Dr. Fotinos Panagakos (Ed.), ISBN: 978-953-307-376-7, InTech, Available from:

http://www.intechopen.com/books/gingival-diseases-their-aetiology-prevention-and-treatment/etiology-ofgingivitis

\section{INTECH}

open science | open minds

\author{
InTech Europe \\ University Campus STeP Ri \\ Slavka Krautzeka 83/A \\ 51000 Rijeka, Croatia \\ Phone: +385 (51) 770447 \\ Fax: +385 (51) 686166 \\ www.intechopen.com
}

\author{
InTech China \\ Unit 405, Office Block, Hotel Equatorial Shanghai \\ No.65, Yan An Road (West), Shanghai, 200040, China \\ 中国上海市延安西路65号上海国际贵都大饭店办公楼 405 单元 \\ Phone: +86-21-62489820 \\ Fax: $+86-21-62489821$
}


(C) 2011 The Author(s). Licensee IntechOpen. This chapter is distributed under the terms of the Creative Commons Attribution-NonCommercialShareAlike-3.0 License, which permits use, distribution and reproduction for non-commercial purposes, provided the original is properly cited and derivative works building on this content are distributed under the same license. 